\title{
BIBECHANA
}

A Multidisciplinary Journal of Science, Technology and Mathematics ISSN 2091-0762 (Print), 2382-5340 (0nline)

Journal homepage: http://nepjol.info/index.php/BIBECHANA

Publisher: Research Council of Science and Technology, Biratnagar, Nepal

\section{Study of far infrared cavity around a post AGB star under IRAS survey at galactic latitude $-3^{0}$}

\author{
A. K. Gautam*, B. Aryal** \\ Central Department of Physics, T.U., Kirtipur,Nepal \\ Email: arjungautamnpj@gmail.com*, baryal@tucdp.edu.np ${ }^{* *}$ \\ Article history: Received 20 February, 2018; Accepted 3 September, 2018 \\ DOI: http://dx.doi.org/10.3126/bibechana.v16i0.20960 \\ This work is licensed under the Creative Commons CC BY-NC License. \\ https://creativecommons.org/licenses/by-nc/4.0/
}

\section{c) (7) (8)}

\section{Abstract}

A systematic search of dust structure in the far infrared $(100 \mu \mathrm{m}$ and $60 \mu \mathrm{m})$ under Infrared Astronomical Satellite (IRAS) survey was performed using Sky View virtual Observatory. In order to find the possible candidate of cavity structure not yet studied, we used SIMBAD database to locate discrete sources in the region. A new relatively symmetric spherical cavity like structure (size: $44.4 \mathrm{pc}$ x 19.4 pc) at R.A.(J2000) $=08^{\mathrm{h}} 03^{\mathrm{m}} 01.65^{\mathrm{s}}$, Dec. $(\mathrm{J} 2000)=-36^{0} 35^{\prime} 47.9^{\prime \prime}$ was found at the distance of about $2800 \mathrm{pc}$. In this article, we have calculated dust color temperature, dust mass and size. We also studied the flux density variation and then calculated temperature and mass profile of the dust of pAGB star using data reduction software Aladin2.5 and Aladin8.0. We have studied a cavity like structure centered at R.A.(J2000) $=08^{\mathrm{h}} 04^{\mathrm{m}} 07.21^{\mathrm{s}}$, Dec.(J2000) $=-37^{0} 11^{\prime} 48.0^{\prime \prime}$. The dust color temperature is found to lie in the range $21.6 \pm 0.09 \mathrm{~K}$ to $22.5 \pm 0.05 \mathrm{~K}$ with an offset of $0.9 \mathrm{~K}$. Such low off set suggests that the post AGB is in local thermodynamic equilibrium. The total mass of the dust in the cavity structure is found about $5.93 \times 10^{25} \mathrm{Kg}(0.00003 \mathrm{M} \odot)$

Keywords: AGB Stars; dust color temperature; dust mass; post AGB star; inclination angle.

\section{Introduction}

Low-to-intermediate mass stars end their life on the asymptotic giant branch (AGB) star. AGB stars are the main distributors of dust into the interstellar medium due to their high mass loss rates in combination with an effective dust condensation. It is therefore important to understand the dust formation process and sequence in their extended atmosphere [1]. An evolutionary phase in which the star sheds most of its mantle into the circumstellar environment through a stellar wind. This stellar wind expands at relatively low velocities and enriches the interstellar medium with elements newly made in the stellar interior. The physical processes controlling the gas and dust chemistry in the outflow, as well as the driving mechanism of the wind itself, are poorly understood and constitute the broader context. When helium in the core is used up, energy production shifts to helium burning in a 
shell. The outer layers of the star expand. The star evolves towards the early-asymptotic giant branch (AGB) and becomes a red-giant for the second time. The expansion of the outer layer extinguishes the hydrogen-burning shell in the intermediate-mass stars, which causes the convective envelope to move inward for a second time. This second dredge-up brings the hydrogen burning products, mainly helium and nitrogen, to the surface but it does not occur in low mass stars since the hydrogen-burning shell is not extinguished.

Following the helium exhaustion in the shell, the hydrogen-burning shell takes over until enough helium has been produced. When the helium shell is sufficiently massive, it will re-ignite and new helium-burning will control the evolution. The helium-burning shell is known to be thermally unstable and it undergoes thermal pulses recurrently. This is the thermally-pulsing AGB (TP-AGB) phase, characterised by two nuclear burning shells surrounding an electron-degenerate carbon-oxygen core and a deep convective envelope. According to [1], thermal pulses may occur in both low- and intermediate mass stars and huge amounts of energy are released. This allows envelope convection to penetrate to the inter-shell region and mix carbon and oxygen enriched material to the surface (third dredge-up). It is important to note that mixing events occurring during these thermal pulses are responsible for the transport of carbon and s-process elements to the surface [2]. It is also equally important to note that observed abundances of post-AGB stars are mostly determined during this stage of mixing episodes driven by consecutive thermal pulses.

During AGB evolution, the helium-burning shell is thermally unstable and causes energy bursts called thermal pulses or flashes repeating apparently every 105 years leading to the development of a convective zone in the shell (3). The phase where hydrogen-shell burning dominates is known as the "inter-pulse phase" which is interrupted by the helium-shell instabilities. The thermal pulse drives a convection zone between the helium and hydrogen burning shells. The fusion products of the heliumburning shell including $12 \mathrm{C}$ and some $16 \mathrm{O}$ are mixed throughout this region. Once a thermal pulse has occurred, a huge amount of energy is released.

Low to intermediate-mass stars cross the HR diagram horizontally from the tip of the asymptotic giant branch (AGB) to the planetary nebula (PN) region after they terminate a rapid mass-loss phase. This transition phase is called post-AGB phase of evolution. From an analysis of the IRAS point source catalog, several post-AGB stars have been identified $[4,5]$. The spectral energy distribution (SED) of post-AGB stars is double peaked. One peak is at far-infrared wavelengths due to the cold dust shell $(100-200 \mathrm{~K})$ and the other peak is at shorter wavelengths, optical or near-infrared, from the obscured central star. The cold dust-shell was observed by IRAS. Most of the post-AGB stars, proto-planetary nebulae (PPNe) and PNe were found within the IRAS color box defined by $\mathrm{F}(12 \mu \mathrm{m}) / \mathrm{F}(25 \mu \mathrm{m})<0.3$ and $\mathrm{F}(25 \mu \mathrm{m}) / \mathrm{F}(60 \mu \mathrm{m})>0.3[6]$.

In this paper, we study the physical properties of a far infrared cavity, that we investigated during a systematic search on IRAS maps, located close to a carbon-rich post AGB star (PAGB 08-36) at -3o galactic latitude. In section 2, we describe methods of calculation. A brief description of the result and discussion will be given in the section 3. Finally, we conclude our results in the section 4.

\section{Methods}

We investigated a cavity-like structure in both 60 and 100 micron IRAS maps around a p-AGB star. Fig.1(a), and 1(b) are cavity images at 60 and $100 \mu \mathrm{m}$ whereas 1(c) is its contour map. We briefly 
describe a method for calculation of dust color temperature and dust mass of the dusty environment around carbon-rich post Asymptotic Giant Branch named p-AGB08-36[7].

\subsection{Dust Color Temperature}

For the calculation of dust color temperature, we adopt the method proposed by Schnee et al. (2005) [8] and Dupac et al. (2003)[9]. According to Schnee et al. (2005)[8], dust color temperature of the emission at a wavelength $\lambda \mathrm{i}$ is given by

$$
\begin{aligned}
& T_{d}=\frac{-96}{\ln \left\{R \times 0.6^{(3+\beta)}\right\}} \\
& \text { where, } \quad \mathrm{R}=\frac{\mathrm{F}(60 \mu \mathrm{m})}{\mathrm{F}(100 \mu \mathrm{m})}
\end{aligned}
$$
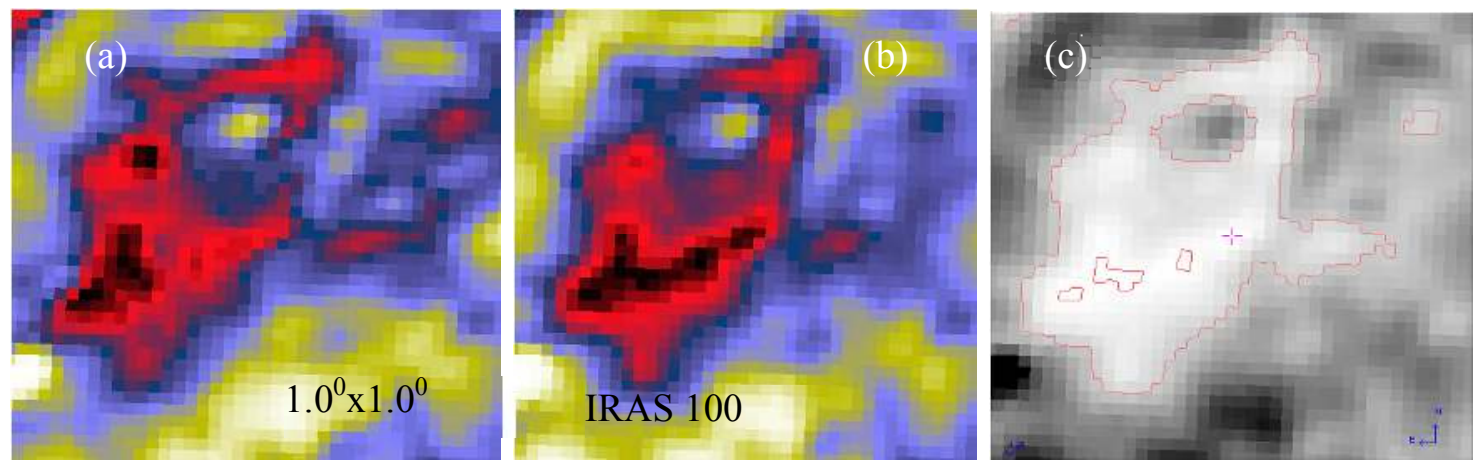

Fig. 1: (a) \& (b) IRAS $60 \mu \mathrm{m}$ and $100 \mu \mathrm{m}$ far infrared image of the core region of AGB 08-36 centered at R.A. $(\mathrm{J} 2000)=08^{\mathrm{h}} 04^{\mathrm{m}} 07.21^{\mathrm{s}}$ and Dec.(J2000) $=-37^{0} 11^{\prime} 48.0^{\prime \prime}$, and (c) Contour map of the cavity where contour level is $1-50$.

$\mathrm{F}(60 \mu \mathrm{m})$ and $\mathrm{F}(100 \mu \mathrm{m})$ are the flux densities in $60 \mu \mathrm{m}$ and $100 \mu \mathrm{m}$ respectively and Eq. (1) is used for calculation of the dust color temperature. The spectral emissivity index $(\beta)$ depends on dust grain properties like composition, size, and compactness. For a pure blackbody would have $\beta=0$, the amorphous layer-lattice matter has $\beta \sim 1$, and the metals and crystalline dielectrics have $\beta \sim 2$ which is used in our calculations.

\subsection{Planck's Function}

The value of Planck's function depends on the wavelength (frequency), and hence the temperature. Finally it is used to calculate dust mass. In 1900, Planck proposed a relation which is named as Planck's function. According to him, the Planck's function is given by

$$
B(\vartheta, T)=\frac{2 h c}{\lambda^{3}}\left[\frac{1}{\frac{h c}{e^{\lambda K T}}-1}\right]
$$

where, $\mathrm{h}=$ Planck's constant, $\mathrm{c}=$ velocity of light, $v=$ frequency at which the emission is observed, $\lambda=$ wavelength of the radiation and $\mathrm{T}=$ temperature of each pixel.

\subsection{Dust Mass}

For the calculation of dust mass, first we need the value of flux density (Fv) at $100 \mu \mathrm{m}$ maps and we use the expression given by Hildebrand (1983)[10], 


$$
M_{d u s t}=\frac{4 a \rho}{3 Q_{v}}\left|\frac{S_{v} D^{2}}{B(v, T)}\right|
$$

where, weighted grain size $(\mathrm{a})=0.1 \mu \mathrm{m}$, grain density $(\rho)=3000 \mathrm{~kg} \mathrm{~m}^{-3}$, grain emissivity $(\mathrm{Qv})=$ 0.0010 (for $100 \mu \mathrm{m}$ ) [11]. So the equation (3) reduces to

$$
M_{\text {dust }}=0.4\left|\frac{S_{v} D^{2}}{B(v, T)}\right|
$$

We use equation (4) to calculate dust mass of the cavity.

\subsection{Inclination angle}

The long axis of KK-loops can be assumed to be inclined by a certain angle with respect to the plane of the sky. The inclination angle i (angle between the line-of-sight and the normal vector of the plane of the loops) can be estimated using Holmberg (1946) [12] formula:

$$
\operatorname{Cos}^{2} i=\frac{\frac{b^{2}}{a^{2}}-q^{2}}{1-q^{2}}
$$

where $\mathrm{b} / \mathrm{a}$ is the measured axial ratio and $\mathrm{q}$ is the intrinsic flatness of the cavity. We use the value of intrinsic flatness $q=0.33$ as suggested by Holmberg (1946) for oblate spheroid structure.

\section{Results and Discussion}

\subsection{Structure: Contour Maps}

While going through the systematic search on IRAS maps, we found an isolated cavity in the $100 \mu \mathrm{m}$ and $60 \mu \mathrm{m}$ at R.A. $(\mathrm{J} 2000)=08^{\mathrm{h}} 04^{\mathrm{m}} 07.21^{\mathrm{s}}$ and Dec. $(\mathrm{J} 2000)=-37^{0} 11^{\prime} 48.0^{\prime \prime}$. With the help of the software ALADIN2.5, we have studied physical properties (size, dust color temperature, dust mass, etc) of the cavity. We selected contour level in such a way that it circles the cavity. The contour map in the contour level 1-50 is shown in the fig.1(c).

\subsection{Flux Density and its Variation}

By using ALADIN 2.5 software, the values of flux densities at $60 \mu \mathrm{m}$ and $100 \mu \mathrm{m}$ have measured. The flux density distribution within the contour of the region of interest has studied. We plotted a graph between flux at $100 \mu \mathrm{m}$ and $60 \mu \mathrm{m}$ with the help of ORIGIN 5.0 which is shown in fig.2(a). From the linear fit, slope of the line was 0.2. The linear equation of the fitted line is, $y=-1.9+0.2 x$. Using the slope of best fitted plot, dust color temperature is found as $23 \mathrm{~K}$ which is slightly more than our calculated value.

Again distribution of flux at $100 \mu \mathrm{m}$ of the pixels within the contour level with right ascension (R.A.) and declination (Dec.) are plotted by using ORIGIN 8.0 and the graph is shown in fig.2(b). Graph shows that all the fluxes from minimum to maximum lie within the contour level. Most of the maximum flux regions lie at the boundary.

\subsection{Dust color Temperature and its Variation}

Using the method of [8], we calculated dust color temperature of each pixel inner the outer isocontour in the region of interest. We use the IRAS $100 \mu \mathrm{m}$ and $60 \mu \mathrm{m}$ FITS images downloaded from the IRAS server. For the calculation of temperature we choose the value of $\beta=2$ following the explanation given by [9]. Variation of temperature with corresponding R.A.(J2000) and Dec.(J2000) are plotted by using ORIGIN 8.0 and the graph is shown in figure 3(a). Graph shows that temperature distributions are in separate cluster but minimum temperature region is little bit shifted from minimum flux density which is unusual behaviour. Such type of nature is obtained due to external factors. Graph 3(b) is the Gaussian fit between dust color temperature and number of pixels with 
offset temperature $0.98 \mathrm{~K}$. This Gaussian fit more or less follow the Gaussian distribution with positive skewness.
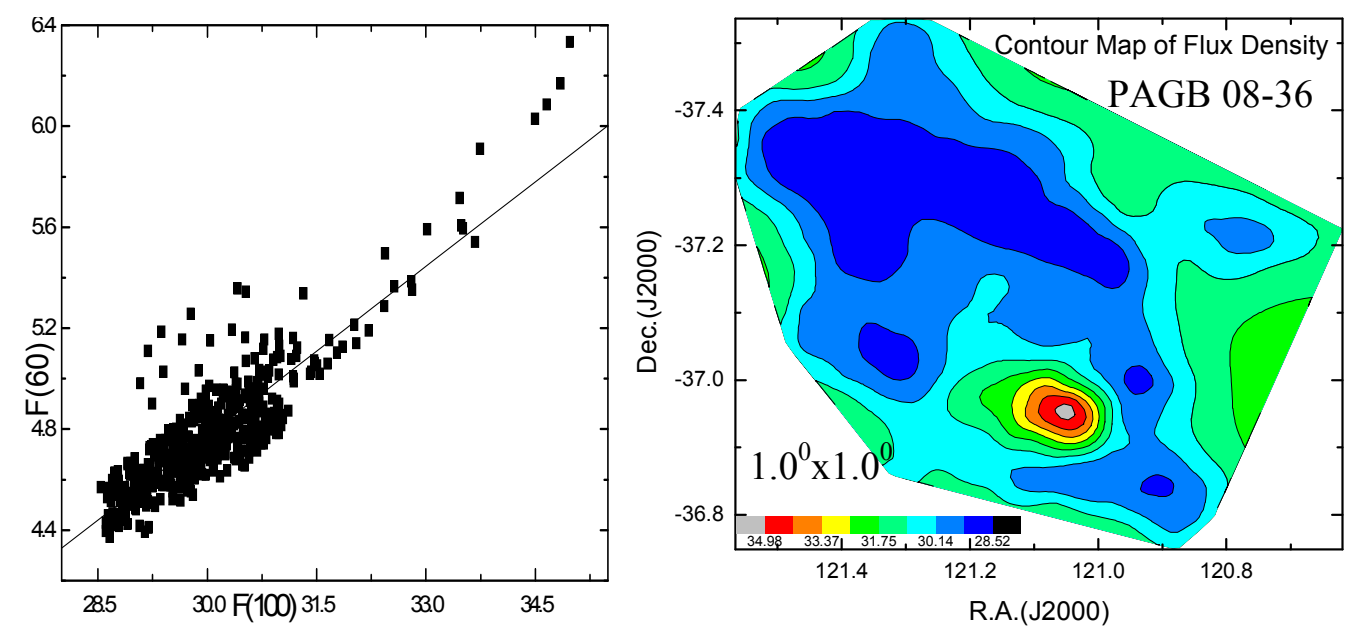

Fig.2(a): The $100 \mu \mathrm{m}$ verses $60 \mu \mathrm{m}$ flux density in the region of interest and 2(b) Contour map at $100 \mu \mathrm{m}$ flux density where the AGB star is located at the center R.A. $(\mathrm{J} 2000)=08^{\mathrm{h}} 04^{\mathrm{m}} 07.21^{\mathrm{s}}$ and Dec. $(\mathrm{J} 2000)=-37^{0} 11^{\prime} 48.0^{\prime \prime}$.
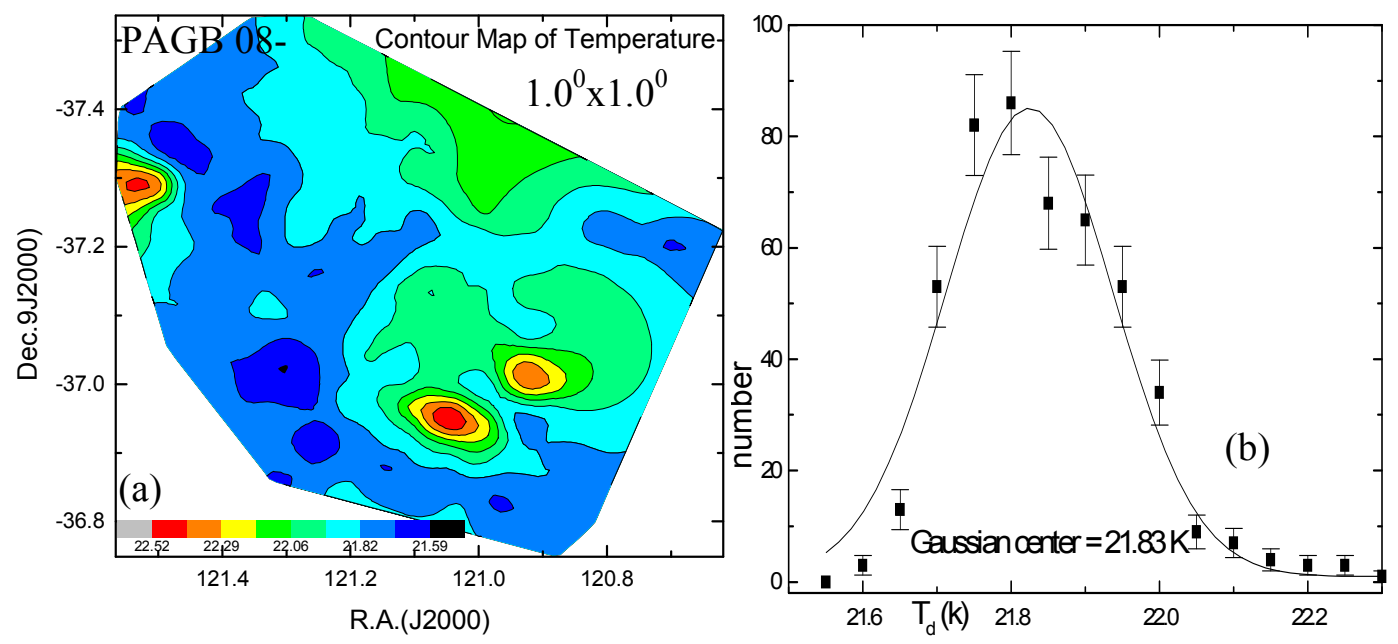

Fig. 3: (a) Contour map of dust color temperature and (b) Gaussian fit between dust color temperature and number of pixels. The field is centered at R.A. $(J 2000)=08^{\mathrm{h}} 04^{\mathrm{m}} 07.21^{\mathrm{s}}$ and Dec. $(\mathrm{J} 2000)=-37^{0} 11^{\prime} 48.0^{\prime \prime}$.

The region in which minimum and maximum temperature is found in the range of $21.6 \mathrm{~K}$ to $22.5 \mathrm{~K}$ with an offset temperature of dust $0.9 \mathrm{~K}$. It suggests that particles are independently vibrating. The cavity may be in thermally pulsating phase. When this result is compared with the result obtained in [13] where temperature variation is $20 \mathrm{~K}$ to $22 \mathrm{~K}$ so our result is also comparable with that result. In the contour map, minimum flux and minimum temperature region are shifted which is due to some 
external factors possibly due to AGB wind. There is good agreement in case of temperature in the Gaussian fit with offset $0.98 \mathrm{~K}$.

\subsection{Size of the Structure}

Major and minor diameter of the structure can be easily calculated by using a simple expression i.e., $\mathrm{L}$ $=\mathrm{R} \times \theta$, where $\mathrm{R}=2.8 \mathrm{kpc}$ is the distance of the structure [7 ] and $\theta=$ pixel size (in radian). After calculation the major and minor diameter of the cavity region are found to be $44.4 \mathrm{pc}$ and $19.4 \mathrm{pc}$ respectively. Thus, the size of the structure is $44.4 \mathrm{pc} \times 19.4 \mathrm{pc}$.

\subsection{Dust Mass Estimation and its variation}

For the calculation of dust mass, we need the distance to the region of interest. The distance of the structure is $2.8 \mathrm{kpc}$ [7]. By using the temperature of each pixel and corresponding distance of the structure, we calculated mass of each pixel. Average mass of each pixel is $8.7 \times 10^{27} \mathrm{~kg}$ and total mass of the structure is $4.3 \times 1030 \mathrm{~kg}$ i.e $2.16 \mathrm{M} \odot$.. But mass of dust obtained around white dwarf WD $1003-44$ in [12] is $0.08 \mathrm{M} \odot .$. It means mass of dust around AGB Star is less than White Dwarf.
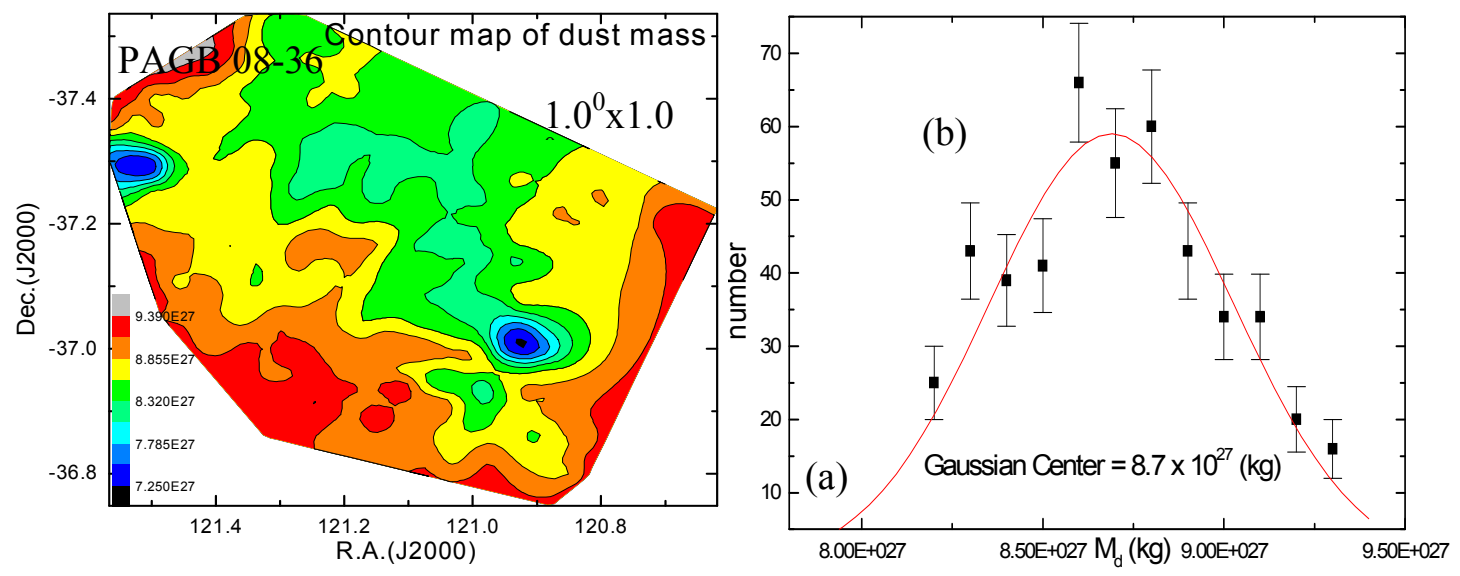

Fig.4: (a) Contour map of dust mass and (b) Gaussian fit between mass and number of pixels. The field is centered at R.A. $(\mathrm{J} 2000)=08^{\mathrm{h}} 04^{\mathrm{m}} 07.21^{\mathrm{s}}$ and Dec. $(\mathrm{J} 2000)=-37^{0} 11^{\prime} 48.0^{\prime \prime}$.

Distribution of dust mass of the pixels within the selected contour level with R.A. (J2000) and Dec.(J2000) are plotted in contour map by using ORIGIN 8.0. Graph obtained is shown in fig.4(a) which shows that minimum temperature region is massive which suggests that it follows cosmological principle means distribution of dust mass is homogeneous and isotropy. Graph 4(b) is the Gaussian fit between mass and number of pixels with standard error $\pm \sigma$. Fitted Gaussian fit of dust mass follow the Gaussian distribution and suggests symmetric behaviour.

\section{Conclusion}

The physical properties of the cavity-like structure that we investigated while searching an effect of AGB wind around carbon-rich AGB stars. A study of flux density and dust color temperature maps, mass of dust, size of the cavity and inclination angle was calculated. Our conclusions are as follows:

- The major and minor diameter of the cavity like structure was found to be $44.4 \mathrm{pc}$ and $19.4 \mathrm{pc}$ respectively so size of the cavity is $44.4 \mathrm{pc} \times 19.4 \mathrm{pc}$. 
- The maximum temperature $22.5 \pm 0.05 \mathrm{~K}$ was found at R.A.(J2000) $=121.040$ \& Dec.(J2000) $=$ 36.940 and minimum temperature $21.6 \pm 0.09 \mathrm{~K}$ was found at R.A.(J2000) $=121.290 \&$ Dec. $(\mathrm{J} 2000)=-36.980$ with offset of $0.9 \mathrm{~K}$. The small value of dust color temperature in the cavity suggests a continuous process by which cavity is supposed to be formed. Low offset in the temperature hints that there is symmetric outflow or symmetric distribution of density and temperature.

- In general, minimum flux and minimum temperature lie at same point in the pixel and in this case nearly normal condition is achieved. Similarly maximum temperature and minimum mass region lie nearly at same region which is normal behavior. It means their distribution follow cosmological principle

- Average mass of each pixel is $8.7 \times 10^{27} \mathrm{~kg}$ and total mass of the cavity is $4.3 \times 10^{30} \mathrm{~kg}$.

- The inclination angle (i) of the cavity is found to be and 840 suggesting that the cavity will be in face-on $(i=0 \circ)$ when it is rotated through 60 from northern to southern direction (it is assumed that the cavity is in the sky plane).

We intend to study the role of cabon-rich PAGB star to form the far-infrared cavity in the future.

\section{Acknowledgements}

We are grateful to the Department of Astro-Particle Physics, Innsbrck University, specially to Prof. R. Weinberger for invoking us to work on dusty environments around AGB stars. This research has made use of SkyView Virtual Observatory, Aladin v2.5 and NASA/IPAC Extragalactic Database (NED). One of the authors (AKG) acknowledges Central Department of Physics, T.U., Nepal for providing various support of Ph.D.

\section{References}

[1] F. Herwig, Evolution of Asymptotic Giant Branch Stars. ARAA 43 (2005) 435. doi.org/10.1146/annurev.astro.43.072103.150600.

[2] Jr. Icko Iben and A.Renzini, Asymptotic giant branch evolution and beyond, Annual review of Astronomy and Astrophysics 21 (983) 271.

[3] A. I. Karakas, J. C. Lattanzio and O. R. Pols, Parameterising the third dredge- up in asymptotic giant branch stars, Astron. Soc. Aust. 19 (2002) 515.

[4] M. Pathasarathy \& S. R. Pottasch, The infrared (IRAS) excess in HD161796 and related stars, A \& A 154 (1986) L16.

[5] S. R. Pottasch, C. bignell, R. Olling \& Zijlstra, Planetary nebula near the galactic plane, A \& A 225 (1989) 521.

[6] W.E.C.J. Van der Veen \& H.J. Habing, The IRAS two color diagram as a tool for studying late stages of stellar evolution, A \& A 194 (1988) 125.

[7] R. Szczerba, N. Siodmiak, G. Stasinska and J.Borkowski, An evolutionary catalogue of galactic post-AGB and related objects, Astronomy and astrophysics 469 (2007) 799. doi.org / 10.1051/0004-6361:20067035.

[8] S. L. Schnee, N. A. Ridge, A. A. Goodman, G. L. Jason, A Complete Look at the Use of IRAS Emission Maps to Estimate Extinction and Dust Temperature, APJ 634 (2005) 442. doi.org/10.1086/511054.

[9] X. Dupac, J. P. Bernard, N. Boudet, M. Giard, J. M. Lamarre, C. Meny, F. Pajot, I. Ristorcelli, G. Serra, B. Stepnik, J. P. Torre, Inverse Temperature Dependance of the Dust Submillimeter Spectral Index, A\&A, .404 (2003) L11. doi.org/10.1051/0004-6361:200030575.

[10] R. H. Hildebrand, The determination of cloud mass and dust characteristics from sub millimeter thermal emission, Q.Jl.R.Astr. Obs.ser 24 (1983) 267. 
[11] K. Young, T. G. Phillips, G. R. Knapp, Circumstellar Shells Resolved in IRAS Survey Data II Analysis, ApJ 409 (1993) 725.

[12] Erik Holmberg, Investigations of the systematic errors in the apparent diameters of the nebulae, MeLus, 117 (1946) $3 \mathrm{H}$.

[13] B. Aryal, R. Weinberger, Dust structure around White Dwarf WD 1003-44 in 60 and $100 \mu \mathrm{m}$ Iras Survey, The Himalayan Physics II (2011) 5. 